\title{
Effects of Numerical Methods on Residual Stress Evaluation by the Incremental Hole-Drilling Technique Using the Integral Method
}

\author{
B. Gore ${ }^{1, a^{*}}$ and J.P. Nobre ${ }^{2, b}$ \\ School of Mechanical, Industrial and Aeronautical Engineering, University of the Witwatersrand, \\ Johannesburg, Private Bag 3, WITS 2050, South Africa \\ abronwyn.gore@students.wits.ac.za, bjoao.nobre@wits.ac.za
}

Keywords: Residual Stress, Incremental Hole-Drilling, Integral Method, Numerical Methods

\begin{abstract}
The incremental hole-drilling technique with Integral Method is widely used to estimate residual stresses. However, the fundamental nature of the Inverse Problem leads to numerically unstable results, which makes stress calculations very sensitive to measurement errors. The extent of this sensitivity is investigated by comparing different combinations of numerical methods for interpolating the input data and solving the equations. The methods are tested on both an experimental and simulated hole-drilling procedure to isolate inaccuracies due to numerical illconditioning and experimental errors. In the experiment, residual stresses determined by X-ray diffraction on a shot-peened sample were taken as the reference for comparison purposes. It was found that interpolating experimental data provides more stable results. Tikhonov Regularisation was more stable than direct methods, but susceptible to numerical errors introduced by the interpolation of input data.
\end{abstract}

\section{Introduction}

With current advancements in computing technology it is now possible to calculate residual stress more accurately. The complex nature of residual stress makes its quantification particularly difficult. The incremental hole-drilling technique is a well-established and widely accepted measurement method, yet it is limited by its sensitivity to errors - this is a fundamental drawback of the holedrilling technique itself and not the associated calculation methods [1,2].

The incremental hole-drilling technique involves the drilling of a small, shallow hole in a number of increments while a strain gauge rosette measures changes in strain at the surface. Calculation procedures can be used to relate the measured strain to relieved stress. Even after simplifying assumptions have been applied there is still no closed-form solution for the residual stress calculation [3]. The fundamental nature of the Inverse Problem always leads to numerically unstable results, which makes stress calculations sensitive to measurement errors. Since its numerical solution is illconditioned, small errors in the input data cause large errors in the results [4]. As with all relaxation methods, residual stress can never be measured exactly where it is being relieved so there is always, by nature, an error in measurements. As the hole depth increases, the amount of relieved stress which can be sensed at the surface tends to zero independently of the residual stress magnitude existing in the deepest layers [5]. Therefore, for a depth equal to the hole diameter, strain relaxation cannot be measured anymore. Regarding the Integral Method in particular, numerically speaking, the stress calculation becomes strongly ill-conditioned for depths greater than half the hole diameter.

The Integral Method is the most widely used calculation method and the only one considered by the ASTM E 837 standard. Despite its sensitivity to errors, it is theoretically the most correct method since it considers that the strain relief at the material's surface is the accumulated result of the residual stresses originally existing in the zone of each successive increment. Therefore, the Integral 
Method is the only one able to give accurate results if strong stress gradients exist [2]. According to the approach proposed by Schajer [2], which was accepted by the ASTM standard [3], the strains and stresses can be divided into hydrostatic and shear components and the residual stress can be determined for isotropic, linear elastic materials, solving the system of linear equations, Eq. 1, Eq. 2 and Eq. 3 - below. This takes into account the fact that the change in strain readings measured at the surface are due to both relieved stress and change in hole geometry.

$$
\begin{aligned}
& \overline{\mathbf{a}} \mathbf{P}=\frac{E}{1+v} \mathbf{p} . \\
& \overline{\mathbf{b}} \mathbf{Q}=E \mathbf{q} . \\
& \overline{\mathbf{b}} \mathbf{T}=E \mathbf{t} .
\end{aligned}
$$

Where:

$\overline{\mathbf{a}}$ is the coefficient matrix of the strain relaxation per unit depth caused by unit normal stress at each depth increment;

$\overline{\mathbf{b}}$ is the coefficient matrix of the strain relaxation per unit depth caused by unit shear stress at each depth increment;

$E$ is the Young's modulus;

$v$ is Poisson's ratio;

$\mathbf{p}, \mathbf{q}$, and $\mathbf{t}$ are the strain components arrays, corresponding to the strains measured by a standard three elements strain-gauge rosette (1,2 and 3 directions) that, for each depth increment, are given by:

$$
\left\{\begin{array}{c}
p=\frac{\varepsilon_{3}+\varepsilon_{1}}{2} \\
q=\frac{\varepsilon_{3}-\varepsilon_{1}}{2}, \\
t=\frac{\varepsilon_{3}+\varepsilon_{1}-2 \varepsilon_{2}}{2}
\end{array}\right.
$$

$\mathbf{P}, \mathbf{Q}$, and $\mathbf{T}$ are the stress components arrays to be calculted that, for each depth increment, are given by (directions 1, 2 and 3 are illustrated in Fig. 1):

$$
\left\{\begin{array}{c}
P=\frac{\sigma_{3}+\sigma_{1}}{2} \\
Q=\frac{\sigma_{3}-\sigma_{1}}{2} . \\
T=\tau_{13}
\end{array}\right.
$$

Since the stress arrays, $\mathbf{P}, \mathbf{Q}$ and $\mathbf{T}$ have been calculated, the principal residual stresses and their directions, for each depth increment, can be determined by:

$$
\left\{\begin{array}{c}
\sigma_{\max }=P+\sqrt{Q^{2}+T^{2}} \\
\sigma_{\min }=P-\sqrt{Q^{2}+T^{2}} \\
\beta=\frac{1}{2} \arctan \frac{-T}{-Q}
\end{array}\right.
$$


In this research, different methods for solving the Integral Method are applied and compared. The methods are applied to a simulated and an experimental hole-drilling procedure. The experiment was performed on a shot-peened steel sample. Results obtained from the developed numerical methods were compared to results from X-ray diffraction as well as the commercially-available software HDRILL (which also uses the Integral method), developed by G. Schajer from the University of British Columbia. Even though the X-ray diffraction method has its own inaccuracies, it was determined accurate enough in a roughly-isotropic shot-peened sample. [6]

\section{Numerical and Experimental Procedure}

To solve the equations set out by the Integral Method, all the matrices must correspond to the same set of hole depth increments. However, the hole-drilling procedure will result in a set of data corresponding to experimental hole depth increments and not the standard increments used in the coefficient matrix determination. One of these sets of data will have to be interpolated to correspond to the other. In this research, a comparison was made between the final residual stress results, depending on which set (experimental or standard data) was interpolated. Once all the input data is in the correct form, the system of matrix equations can be solved. In this preliminary work, this was done using one of two numerical methods: Gauss-Seidel method or Tikhonov Regularisation.

Interpolating Standard Data. Bivariate Interpolation is a method of interpolating functions of more than one variable. It is recommended for this particular application by Schajer [2]. The coefficient matrices can be seen as tabulations of selected values of functions of hole depth and stress depth. Hole depth is the total depth of the hole at that increment and stress depth is the depth of the relieved strain component under consideration. Physically, stress depth always has to be less than or equal to hole depth which creates a lower triangular coefficient matrix. This feature requires that a triangular scheme of the Bivariate Interpolation be adopted. Using Bivariate Interpolation, the standard coefficient matrices can be interpolated to correspond to the experimental hole depth increments.

Interpolating Experimental Data. Each of the three strain measurements $\varepsilon_{1}, \varepsilon_{2}$ and $\varepsilon_{3}$ are plotted with hole depth and a cubic spline is calculated for each set. Cubic spline interpolation is chosen over polynomial interpolation because polynomial interpolations tend to oscillate for many data points whereas cubic splines do not. Cubic splines, therefore, give much smoother interpolating functions, especially if there are abrupt changes in the data. Cubic splines have been shown to be the more useful and common in engineering practice compared to its linear and quadratic counterparts [7].

Gauss-Seidel Method. This is a direct method for solving matrix equations. Gauss-Seidel is an iterative method so round off errors will be mitigated [7].

Tikhonov Regularisation. This is the most common method for solving ill-conditioned equations and is prescribed by the ASTM standard [3,7].

Using Python 2.7, two scripts were written to process the data before solving the system of equations:

- SDP (Standard Data Processor): standard data is interpolated.

- EDP (Experimental Data Processor): experimental data is interpolated.

Two scripts were written for solving the Integral Method equations once the input data had been processed:

- GSS (Gauss-Seidel Solver): solves the equations using the Gauss-Seidel method.

- $\quad$ TRS (Tikhonov Regularisation): solves the equations using Tikhonov Regularisation

These are combined to create four separate methods: SDP-GSS, SDP-TRS, EDP-GSS and EDPTRS.

Simulated Hole-Drilling Procedure. A finite element analysis of the incremental hole-drilling method was carried out using ANSYS APDL code. A $100 \mathrm{MPa}$ uniform tensile stress was applied to a plate in the $\mathrm{x}$-direction to simulate a constant uniform residual stress state. Changes in strain on the surface were recorded and integrated for the geometry of a standard ASTM B strain gauge rosette. 
The recommended hole depth increments $(0.05 \mathrm{~mm}$ increments $)$ were then removed. Because there were no experimental errors in the simulation, the numerical conditioning of each method could be tested.

Experimental Hole-Drilling Procedure. The methods were then applied to real experimental results of the hole-drilling procedure on a shot-peened piece of steel. X-ray diffraction was also performed on the sample and the results from the hole-drilling procedure were run through H-DRILL and the scripts developed in this research so they could all be compared.

\section{Results}

Simulated Hole-Drilling Procedure. Figure 1 shows the calculated maximum principal residual stress for each numerical method. The root mean square deviations (RMSD) values were $3.73 \mathrm{MPa}$ for SDP-GSS, 1.74 MPa for SDP-TRS, 3.15 MPa for EDP-GSS and 0.88 MPa for EDP-TRS.

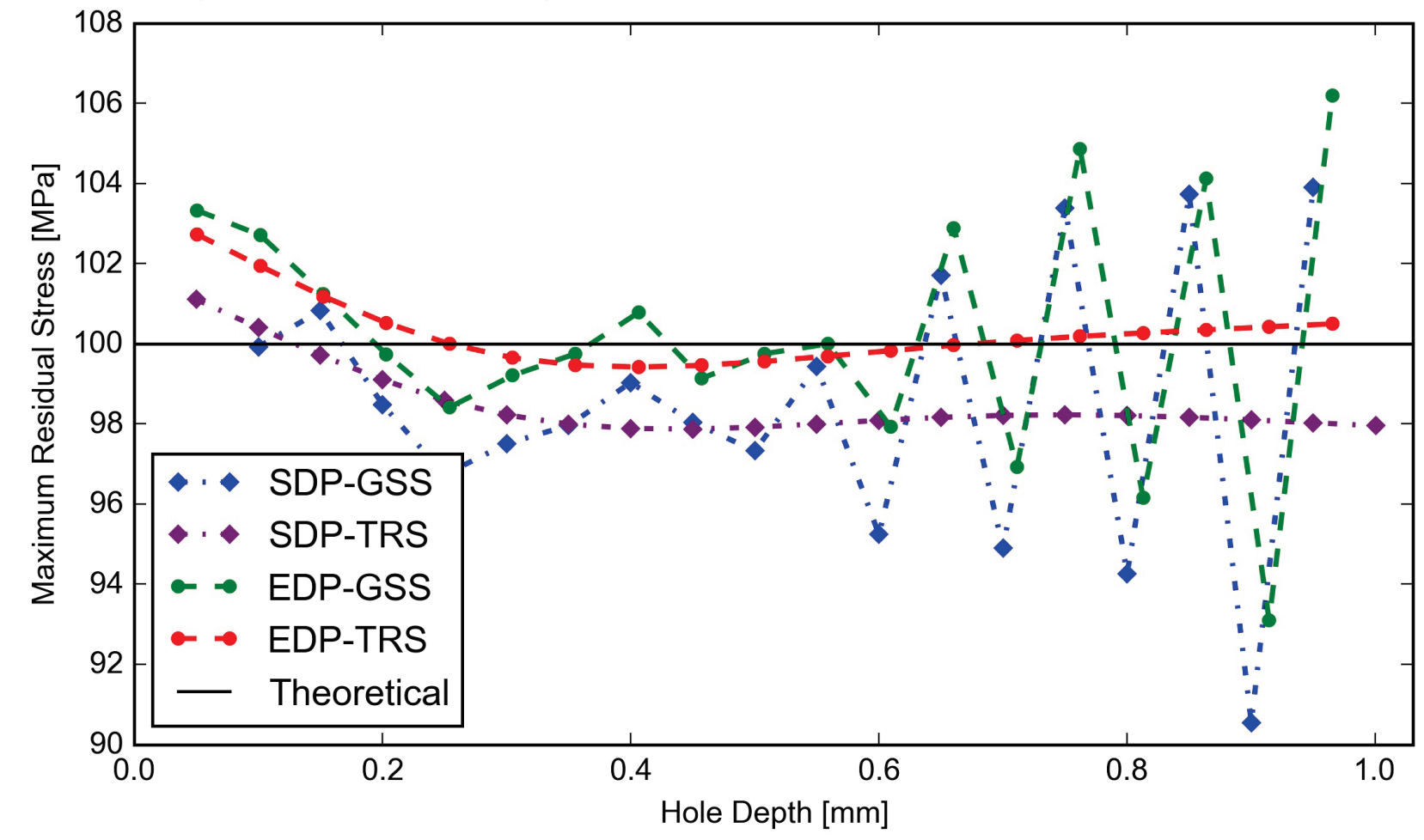

Figure 1 - Maximum Residual Stress for Simulated Hole-Drilling Procedure

Experimental Hole-Drilling Procedure. Figure 2 shows a comparison between the methods developed in this research and the X-ray diffraction results (left) and a comparison between the X-ray diffraction results, H-DRILL and the best performing numerical method EDP-TRS (right).

\section{Discussion}

Simulated Hole-Drilling Procedure. In the simulation, the Gauss-Seidel methods both oscillate about the true value with increased oscillation as hole depth increases. This is because as hole depth increases the problem becomes more numerically ill-conditioned. Because there are 20 hole depth increments, the matrices in the equations are large, which also contributes to ill-conditioning.

The experimental and standard data corresponded to the same hole depth increments so theoretically it shouldn't have mattered which one was interpolated. For the Gauss-Seidel methods it didn't make any difference but it is interesting to note that it made a big difference to the Tikhonov Regularisation method, with the bivariate interpolation introducing significant errors into this method. This suggests that Tikhonov Regularisation has a greater sensitivity to numerical errors in input data. 
There is no accurate way to measure the actual error in these methods because even in the ANSYS simulation the actual residual stress is not known precisely.
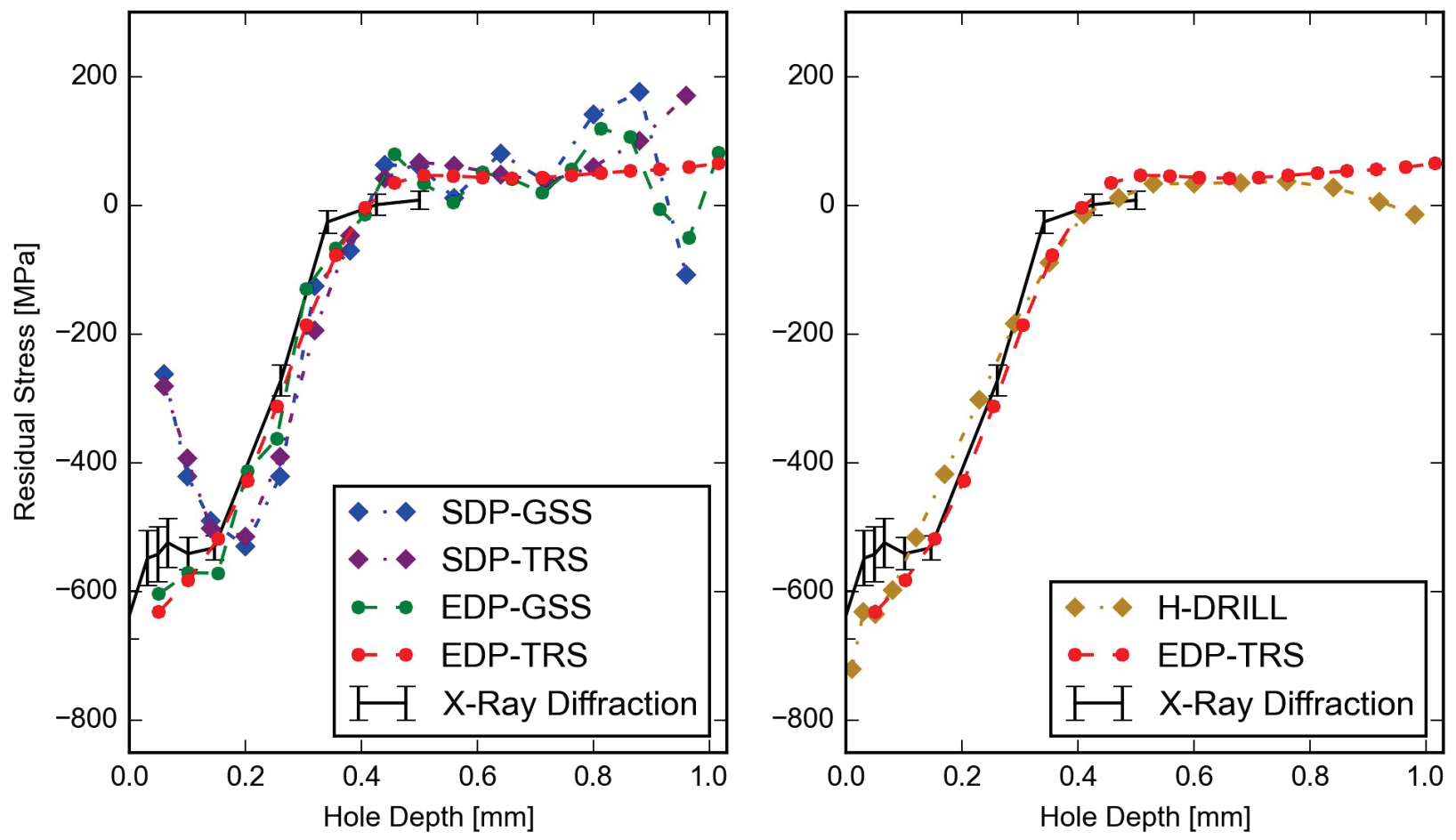

Figure 2 - Residual Stress for Experimental Hole-Drilling Procedure

Experimental Hole-Drilling Procedure. For the shot-peened sample, the ASTM E837 method recommends that if enough stress values have a magnitude greater than $60 \%$ of the yield stress of the material that the calculation is not accurate. In this case only one point falls into that category and since localised increases in yield stress due to the surface treatment has not been taken into account we can neglect it.

We took the X-ray diffraction values as the "true" values although this method also has its own inaccuracies - stresses in only two directions are measured so principal stress cannot be calculated. Although at each step the most compressive stress value was taken and the field is approximately isotropic, there will still be errors in this data.

The levelling off of stress values results in small change of strain measurements at the surface which makes it more sensitive to errors which accounts for the greater amplitude of oscillation with increasing hole depth. In the actual hole-drilling procedure the drill will never drill a perfectly cylindrical hole like in FEM simulation. There will inevitably be a chamfer around the bottom of the hole. This difference in geometry from the FEM used to calculate the coefficient matrices leads to errors in the computed stress. The stress relieved is actually smaller because it is acting on a smaller area (hole diameter is decreased at the chamfer). This causes significant errors at small hole depth increments where the non-cylindrical part of the hole makes up a large percentage of the total hole depth. This could account for the large errors seen at smaller hole depths.

In this procedure a large number of hole depth increments were used (21) which, again, leads to ill-conditioned matrix equations.

From Figure 2 we can see that the experimental data interpolation has consistently closer values. This could be a result of ill numerical condition from the bivariate interpolation. In these results, the experimental data interpolation has similar values for both Gauss-Seidel and Tikhonov Regularisation. The Gauss-Seidel results both oscillate more than the Tikhonov Regularisation 


\section{Summary}

The combination of interpolating experimental data and using a Tikhonov Regularisation method for solving the residual stress profile has consistently produced the best results in terms of accuracy, smoothness and numerical stability. Tikhonov Regularisation makes a significant improvement to results from inputs which are numerically ill-conditioned due to small changes in strain. Interpolating the experimental data as opposed to the standardised data, significantly reduces the propagation of experimental errors and reduces numerical errors.

\section{References}

[1] A. Niku-Lari, J. Lu, J.F. Flavenot, Measurement of Residual-Stress Distribution by the Incremental Hole-Drilling Method, Experimental Mechanics, Vol. 25, No. 2, 1985, pp. 175-185. http://dx.doi.org/10.1007/BF02328809

[2] G.S. Schajer, Measurement of Non-Uniform Residual Stresses Using the Hole-Drilling Method. Part II - Practical Application of the Integral Method, Transactions of the ASME, Vol. 110, No. 4, 1988, Part I: pp. 338-343 and Part II: pp. 344-349.

[3] Determining Residual Stresses by the Hole-Drilling Strain-Gage Method, ASTM Standard E837, 2013.

[4] B. Zuccarello, Optimal Calculation Steps for the Evaluation of Residual Stress by the Incremental Hole-drilling Method, Experimental Mechanics, Vol. 39, No. 2, 1999, pp. 117-124. http://dx.doi.org/10.1007/BF02331114

[5] G.S. Schajer, E. Altus, Stress Calculation Error Analysis for Incremental Hole-Drilling Residual Stress Measurements, Journal of Engineering Materials and Technology, Vol. 118, No. 1, 1996, pp. 120-126. http://dx.doi.org/10.1115/1.2805924

[6] G.S. Schajer, Advances in Hole-Drilling Residual Stress Measurements, Experimental Mechanics, Vol. 50, No. 2, 2010, pp. 159-168. http://dx.doi.org/10.1007/s11340-009-9228-7

[7] S.C. Chapra, R.P. Canale, Numerical Methods for Engineers, fifth ed., McGraw-Hill, Singapore, 2006. 